\title{
EXTREME POINTS OF INTEGRAL FAMILIES OF ANALYTIC FUNCTIONS
}

\author{
KEIKO DOW ${ }^{\otimes}$ and D. R. WILKEN \\ (Received 13 August 2011; accepted 17 October 2012; first published online 14 March 2013) \\ Communicated by P. C. Fenton
}

\begin{abstract}
Extreme points of compact, convex integral families of analytic functions are investigated. Knowledge about extreme points provides a valuable tool in the optimization of linear extremal problems. The functions studied are determined by a two-parameter collection of kernel functions integrated against measures on the torus. For specific choices of the parameters many families from classical geometric function theory are included. These families include the closed convex hull of the derivatives of normalized close-to-convex functions, the ratio of starlike functions of different orders, as well as many others. The main result introduces a surprising new class of extreme points.
\end{abstract}

2010 Mathematics subject classification: primary 30C45; secondary 30E20.

Keywords and phrases: extreme points.

\section{Main theorem}

Let $\mathbb{D}$ and $\Gamma$, respectively, denote the open unit disk and the unit circle in the complex plane $\mathbb{C}$. Let $H(\mathbb{D})$ denote the space of functions analytic in $\mathbb{D}$ and let $\mathbb{T}=\Gamma \times \Gamma$ denote the torus. We investigate extreme points of the compact, convex families in $H(\mathbb{D})$ defined by, for $p, q>0$,

$$
F_{p, q}=\left\{f_{\mu}(z)=\int_{\mathbb{T}} \frac{(1-x z)^{p}}{(1-y z)^{q}} d \mu(x, y): \mu \text { is a probability measure on } \mathbb{T}\right\} .
$$

For $p=1, q=3$, the family $F_{1,3}$ is the closed convex hull of the derivatives of the normalized close-to-convex functions on $\mathbb{D}$ [4]. If $1 \leq p \leq q \leq 2, p=2(1-\beta)$ and $q=2(1-\alpha)$, one obtains the closed convex hull of the ratio of two starlike functions of order $\alpha$ and $\beta$ [1]. The closed convex hull of a number of other families of analytic functions can be identified with specific $F_{p, q}$ families [5, 6]. Solving linear extremal problems reduces to optimizing linear functionals over the extreme points of these

(c) 2013 Australian Mathematical Publishing Association Inc. 1446-7887/2013 \$16.00 
families, which leads to the question of determining, for a given $p$ and $q$, the extreme points of $F_{p, q}$.

For many calculations, it is convenient to make a change of variables: $(x, y) \rightarrow(x y, y)$. Let

$$
k_{x, y}(z)=\frac{(1-x y z)^{p}}{(1-y z)^{q}}=\sum_{n=0}^{\infty} K_{n}(x, y) z^{n} .
$$

With this choice of notation and change of variables,

$$
F_{p, q}=\left\{f_{\mu}(z)=\int_{\mathbb{T}} k_{x, y}(z) d \mu(x, y): \mu \text { is a probability measure on } \mathbb{T}\right\} .
$$

We also define

$$
I_{p, q}=\left\{f_{\mu}(z)=\int_{\mathbb{T}} k_{x, y}(z) d \mu(x, y): \mu \text { is a complex Borel measure on } \mathbb{T}\right\} .
$$

Then $I_{p, q}$ is the linear span of $F_{p, q}$ in $H(\mathbb{D})$.

Consider the curve $C_{p}=\left\{(1-x)^{p}:|x|=1\right\}$ and let $E_{p}$ denote the closed convex hull of $C_{p}$ in $\mathbb{C}$. The following are well-known facts.

(i) Every extreme point of $F_{p, q}$ is a kernel function $k_{x, y}$.

(ii) If $x \neq 1$ and $(1-x)^{p}$ is an extreme point of $E_{p}$, then $k_{x, 1}$ is an extreme point of $F_{p, q}$.

(iii) The family $F_{p, q}$ is closed under rotations. That is, if $f$ is a function in $F_{p, q}$ and $|u|=1$, then $g(z)=f(u z)$ is also in $F_{p, q}$. Any rotation of an extreme point is an extreme point.

By rotation, it follows that $k_{x, y}$ is an extreme point if and only if $k_{x, 1}$ is an extreme point. If $0<p \leq 1$, the curve $C_{p}$ encloses a convex region and every point on $C_{p}$ is an extreme point of $E_{p}$. In this case, by (ii), $k_{x, 1}$ is an extreme point of $F_{p, q}$ whenever $x \neq 1$. For $p>1$, as one traverses the curve $C_{p}$ in either direction starting at $2^{p}(x=-1)$ and ending up at the origin $(x=1)$, there are two distinguished 'turning points'. These turning points occur when $|\arg (x)|=\pi(p-1) /(p+1)$ and correspond to the points on $C_{p}$ where $\operatorname{Re}(1-x)^{p}$ attains its minimum value. In this case, the convex set $E_{p}$ is bounded by part of the curve $C_{p}$ - the part traversed from one turning point through $2^{p}$ to the other turning point - together with the vertical line segment joining the two turning points. Thus, if $p>1$, a point $(1-x)^{p}$ is an extreme point of $E_{p}$ if and only if $\pi(p-1) /(p+1) \leq|\arg (x)| \leq \pi$. Again by (ii), $k_{x, 1}$ is an extreme point of $F_{p, q}$ for $|\arg (x)|$ in this interval and $x \neq 1$.

Two fundamental questions arise. The first question is whether any points $(1-x)^{p}$, with $|\arg (x)|<\pi(p-1) /(p+1)$, yield kernel functions that are extreme points of $F_{p, q}$. The second question is whether the parameter $q$ plays a role in the determination of extreme points. In this paper we address both of these questions. The main theorem answers the first affirmatively and the constructive approach in the proof utilizes the parameter $q$. 
Theorem 1.1 (Main theorem). For $p>1$ and $q>0$ there exist points $(1-x)^{p}$ on $C_{p}$ with $|\arg (x)|<\pi(p-1) /(p+1)$ such that the corresponding kernel functions $k_{x, 1}$ are extreme points of $F_{p, q}$.

\section{Preliminaries}

Fix $p, q>0$. Let $F=F_{p, q}$ and $I=I_{p, q}$. A generalized functional on $F$ is a map $L: F \rightarrow \mathbb{C}$ that is continuous on $F$ and linear on $I$. Let $F^{*}$ denote the collection of all generalized functionals on $F$. If $f \in F$ and there exists $L \in F^{*}$, with $\operatorname{Re} L$ nonconstant on $F$, such that $\operatorname{Re} L(f)=\max _{F} \operatorname{Re} L$, then $f$ is called a generalized support point of $F$. If $f$ uniquely maximizes $\operatorname{Re} L$, then $f$ is called a generalized exposed point of $F$.

REMARK 2.1. The set of generalized support points of $F$ corresponding to a generalized functional $L$ is a compact, convex, extremal subset of $F$. Therefore it contains extreme points of $F$ as a consequence of the Krein-Milman theorem. A generalized exposed point then is an extreme point of $F$.

Our approach is to associate the space of generalized functionals with a subspace of the space $C(\mathbb{T})$ of all continuous functions on $\mathbb{T}$ and to associate generalized support points with points where the real parts of certain functions in this subspace achieve their maximum value.

Let $B=\overline{S p}\left\{K_{n}: n=0,1,2, \ldots,\right\}$, that is, $B$ is the uniformly closed linear span of the coefficient functions $K_{n}(x, y)$ in $C(\mathbb{T})$. If one writes

$$
(1-x y z)^{p}=\sum_{n=0}^{\infty} A_{n}(p) x^{n} y^{n} z^{n} \quad \text { and } \quad \frac{1}{(1-y z)^{q}}=\sum_{n=0}^{\infty} B_{n}(q) y^{n} z^{n}
$$

then

$$
A_{n}(-p)=B_{n}(q) \quad \text { and } \quad K_{n}(x, y)=\sum_{i=0}^{n}\left[A_{i}(p) x^{i} B_{n-i}(q)\right] y^{n}=K_{n}(x, 1) y^{n} .
$$

For example, when $p=2$ and $q=1$,

$$
K_{0}(x, y)=1, \quad K_{1}(x, y)=(1-2 x) y, \quad K_{n}(x, y)=(1-x)^{2} y^{n}, \quad n \geq 2,
$$

and

$$
B=\left\{a_{0}+a_{1}(1-2 x) y+(1-x)^{2} y^{2} f(y): a_{0}, a_{1} \in \mathbb{C} \text { and } f \in A_{0}\right\},
$$

where $A_{0}$ is the classic disk algebra on $\mathbb{D}$. In general, $B$ is not so easily described.

We use the following special case of an unpublished theorem in [7]. For completeness, we sketch the proof.

THEOREM 2.2. $F^{*}$ is isometrically isomorphic to $B$ where, for $L \in F^{*},\|L\|=\sup \{|L(f)|$ : $f \in F\}$. The correspondence is given by $L_{G} \leftrightarrow G$ where, for each $G \in B$ and $f_{\mu} \in I$,

$$
L_{G}\left(f_{\mu}\right)=\int_{\mathbb{T}} G(x, y) d \mu(x, y) .
$$


Proof. Let $G \in B$. First we show that $L_{G}$ is well defined. Suppose that $f_{\mu}=f_{v} \in I$. Then

$$
\int_{\mathbb{T}} k_{x, y}(z) d \mu(x, y)=\int_{\mathbb{T}} k_{x, y}(z) d v(x, y), \quad z \in \mathbb{D} .
$$

Hence

$$
\sum_{n=0}^{\infty} \int_{\mathbb{T}} K_{n}(x, y) d \mu(x, y) z^{n}=\sum_{n=0}^{\infty} \int_{\mathbb{T}} K_{n}(x, y) d v(x, y) z^{n}
$$

and

$$
\int_{\mathbb{T}} K_{n}(x, y) d \mu(x, y)=\int_{\mathbb{T}} K_{n}(x, y) d v(x, y), \quad n=0,1,2, \ldots
$$

Since $G \in B=\overline{S p}\left\{K_{n}: n=0,1,2, \ldots,\right\}, \int_{\mathbb{T}} G d \mu=\int_{\mathbb{T}} G d v$. Thus $L_{G}\left(f_{\mu}\right)=L_{G}\left(f_{v}\right)$ and $L_{G}$ is well defined on $F$. Also, $L_{G}$ is clearly linear on $I$.

To show that $L_{G} \in F^{*}$, it remains to show that $L_{G}$ is continuous on F. Suppose that $f_{\mu_{n}}$ converges to $f_{\mu}$ in $F$. Let $b_{n}=L_{G}\left(f_{\mu_{n}}\right)=\int_{\mathbb{T}} G d \mu_{n}$ and let $b=L_{G}\left(f_{\mu}\right)=\int_{\mathbb{T}} G d \mu$. The set of probability measures on $\mathbb{T}$ is compact, convex and metrizable in the weak topology. Let $\lambda$ be any weak* subsequential limit point of $\left\{\mu_{n}\right\}$. Apply the weak* convergence to $k_{x, y}(z)$ for each fixed $z$ in $\mathbb{D}$ and use the fact that $f_{\mu_{n}}(z) \rightarrow f_{\mu}(z)$, that is, $\int_{\mathbb{T}} k_{x, y}(z) d \mu_{n}(x, y) \rightarrow \int_{\mathbb{T}} k_{x, y}(z) d \mu(x, y)$, to conclude that $\int_{\mathbb{T}} k_{x, y}(z) d \lambda(x, y)=$ $\int_{\mathbb{T}} k_{x, y}(z) d \mu(x, y)$ for $z$ in $\mathbb{D}$. Thus $L_{G}\left(f_{\lambda}\right)=L_{G}\left(f_{\mu}\right)=b$ is the unique subsequential limit point of $\left\{b_{n}\right\}$. Therefore $b_{n} \rightarrow b, L_{G}$ is continuous on $F$ and $L_{G} \in F^{*}$.

Conversely, we show that every $L \in F^{*}$ is of the form given in the theorem. Fix $L$ in $F^{*}$ and let $G(x, y)=L\left(k_{x, y}\right)$. Since $L$ is continuous on $F, G$ is continuous on $\mathbb{T}$. We also need to show that $G \in B$. Suppose that $G \notin B$. Then, by a corollary to the Hahn-Banach theorem, there exists a complex Borel measure $\lambda$ such that $\int_{\mathbb{T}} G d \lambda=1$ and $\int_{\mathbb{T}} h d \lambda=0$ for all $h$ in $B$. For fixed $z \in \mathbb{D}, k_{x, y}(z)=\sum_{n=0}^{\infty} K_{n}(x, y) z^{n}$ and the series converges uniformly on $\mathbb{T}$, so that $k_{x, y}(z) \in \overline{S p}\left\{K_{n}: n=0,1,2, \ldots\right\}=B$. We thus have $f_{\lambda}(z)=\int_{\mathbb{T}} k_{x, y}(z) d \lambda(x, y)=0$ for each $z \in \mathbb{D}$, that is, $f_{\lambda} \equiv 0$. Then

$$
\begin{aligned}
\int_{\mathbb{T}} G(x, y) d \lambda(x, y) & =\int_{\mathbb{T}} L\left(k_{x, y}(z)\right) d \lambda(x, y) \\
& =L\left(\int_{\mathbb{T}} k_{x, y}(z) d \lambda(x, y)\right)=L\left(f_{\lambda}\right)=L(0)=0,
\end{aligned}
$$

contradicting the choice of $\lambda$. Hence $G \in B$ and, if $f_{\mu} \in I$, then

$$
\begin{aligned}
L\left(f_{\mu}\right) & =L\left(\int_{\mathbb{T}} k_{x, y}(z) d \mu(x, y)\right) \\
& =\int_{\mathbb{T}} L\left(k_{x, y}(z)\right) d \mu(x, y)=\int_{\mathbb{T}} G(x, y) d \mu(x, y)=L_{G}\left(f_{\mu}\right) .
\end{aligned}
$$


Finally, to show that the correspondence is isometric,

$$
\begin{aligned}
\left\|L_{G}\right\| & =\sup \left\{\left|L_{G}(f)\right|: f \in F\right\} \\
& =\sup \left\{\left|\int_{\mathbb{T}} G d \mu\right|: \mu \text { is a probability measure on } \mathbb{T}\right\} \\
& \leq\|G\|=\sup \{|G(x, y)|:(x, y) \in \mathbb{T}\} .
\end{aligned}
$$

If $\left(x_{0}, y_{0}\right)$ is such that $\left|G\left(x_{0}, y_{0}\right)\right|=\|G\|$, then $\left|L_{G}\left(k_{x_{0}, y_{0}}\right)\right|=\left|G\left(x_{0}, y_{0}\right)\right|=\|G\|$ and the correspondence is an isometric isomorphism.

A point $\left(x_{0}, y_{0}\right) \in \mathbb{T}$ is a peak point of a closed subspace $B$ of $C(\mathbb{T})$ if there exists $f \in B$ such that $f\left(x_{0}, y_{0}\right)=1$ while $|f(x, y)|<1$ for all $(x, y) \in \mathbb{T}$ such that $(x, y) \neq$ $\left(x_{0}, y_{0}\right)$. A point $\left(x_{0}, y_{0}\right) \in \mathbb{T}$ is a peak point of $\operatorname{Re} B$ if there exists $f \in B$ such that $\operatorname{Re} f\left(x_{0}, y_{0}\right)=1$ while $\operatorname{Re} f(x, y)<1$ for all $(x, y) \in \mathbb{T}$ such that $(x, y) \neq\left(x_{0}, y_{0}\right)$. In each case we say that the function peaks at the point $\left(x_{0}, y_{0}\right)$.

Corollary 2.3. $\left(x_{0}, y_{0}\right)$ is a peak point of $\operatorname{Re} B$ if and only if $k_{x_{0}, y_{0}}$ is a generalized exposed point of $F$.

Proof. Suppose that $\left(x_{0}, y_{0}\right)$ is a peak point of $\operatorname{Re} B$. Then there exists $G$ in $B$ such that $\operatorname{Re} G\left(x_{0}, y_{0}\right)=1$ and $\operatorname{Re} G(x, y)<1$ if $(x, y) \neq\left(x_{0}, y_{0}\right)$.

For any probability measure $\mu$ on $\mathbb{T}$,

$$
\operatorname{Re} L_{G}\left(f_{\mu}\right)=\operatorname{Re} \int_{\mathbb{T}} G(x, y) d \mu(x, y)=\int_{\mathbb{T}} \operatorname{Re} G(x, y) d \mu(x, y) .
$$

If $f_{\mu}=k_{x_{0}, y_{0}}$ and $\mu$ is unit point mass at $\left(x_{0}, y_{0}\right)$, then $\operatorname{Re} L_{G}\left(k_{x_{0}, y_{0}}\right)=1$. If $f_{\mu} \neq k_{x_{0}, y_{0}}$, then $\mu$ is not unit point mass at $\left(x_{0}, y_{0}\right)$ and $\operatorname{Re} L_{G}\left(f_{\mu}\right)<1$. Thus $k_{x_{0}, y_{0}}$ is a generalized exposed point.

Conversely, if $f_{\mu}$ is a generalized exposed point, then $f_{\mu}$ is an extreme point and $f_{\mu}=k_{x_{0}, y_{0}}$ for some $\left(x_{0}, y_{0}\right)$. Suppose that $L$ is such that $\operatorname{Re} L$ peaks at $k_{x_{0}, y_{0}}$. Let $G$ be such that $L=L_{G}$. Then, using unit point mass at $(x, y)$ to represent each kernel function $k_{x, y}$,

$$
\begin{gathered}
1=\operatorname{Re} L_{G}\left(k_{x_{0}, y_{0}}\right)=\operatorname{Re} G\left(x_{0}, y_{0}\right) \quad \text { and } \\
1>\operatorname{Re} L_{G}\left(k_{x, y}\right)=\operatorname{Re} G(x, y), \quad \text { if }(x, y) \neq\left(x_{0}, y_{0}\right) .
\end{gathered}
$$

Hence $\left(x_{0}, y_{0}\right)$ is a peak point of $\operatorname{Re} B$.

The final preliminary result concerns a method for constructing peaking functions for the space $\operatorname{Re} B$. The ideas in the proof originally come from Bishop [2], who was interested in constructing peak points for algebras of functions. A concise statement and proof of Bishop's result is given by [3, Theorem 11.1 in Ch. 2]. Rice [7] adapted the argument to spaces of functions and proved the following theorem.

Theorem 2.4. Let $X$ be a compact metric space, $E$ be a closed subset of $X, B$ be a closed subspace of $C(X)$ and $x_{0} \in E$. Suppose that there exist numbers $c$ and $M$, 
with $0<c<1 \leq M$, such that, for any neighborhood $U$ of $E$ in $X$, there exists $H \in B$ such that:

(a) $\|H\| \leq M$;

(b) $\operatorname{Re} H(x)<c, x \notin U$; and

(c) $\operatorname{Re} H(x)<1=\operatorname{Re} H\left(x_{0}\right)$, for all $x \in E$ with $x \neq x_{0}$.

Then there exists $G \in B$ such that $\operatorname{Re} G\left(x_{0}\right)>\operatorname{Re} G(x)$ for all $x \in X$ with $x \neq x_{0}$.

We need a modified version of this theorem. We give the proof of the modified version, the details of which are guided by the arguments of Bishop [2] and Rice [7].

Theorem 2.5. Let $X$ be a compact metric space, $E$ be a closed subset of $X, B$ be a closed subspace of $C(X)$ and $x_{0} \in E$. Suppose that there exist numbers $c$ and $M$, with $0<c<1 \leq M$, functions $H_{n} \in B, n \in \mathbb{N}$, and $H: X \rightarrow \mathbb{C}$ satisfying:

(a) $\left\|H_{n}\right\| \leq M$ for every $n$;

(b) $H_{n}$ converges uniformly to $H$ on $E$;

(c) $\operatorname{Re} H_{n}\left(x_{0}\right)=1$ for every $n$;

(d) $1=\operatorname{Re} H\left(x_{0}\right)>\operatorname{Re} H(x)$ for all $x \in E$ with $x \neq x_{0}$;

(e) $H_{n}$ converges to $H$ uniformly on compact subsets of $X \backslash E$; and

(f) $\operatorname{Re} H(x)<c, x \in X \backslash E$.

Then, for a given relatively open neighborhood $W$ of $x_{0}$ in $E$, there exists $G \in B$ such that

$$
\left\{x \in X: \operatorname{Re} G(x)=\max _{x^{\prime} \in X} \operatorname{Re} G\left(x^{\prime}\right)\right\} \subseteq W .
$$

Proof. Given any open neighborhood $U$ of $E$, the hypotheses allow one to find $h \in B$ satisfying:

(i) $\|h\| \leq M$;

(ii) $\operatorname{Re} h<c$ on $X \backslash U$;

(iii) $\operatorname{Re} h\left(x_{0}\right)=1$; and

(iv) $\operatorname{Re} h<1$, on $E \backslash W$.

Let $\left\{U_{n}\right\}_{n=0}^{\infty}$ be a decreasing sequence of neighborhoods of $E$ with $U_{0}=X$ and $E=\bigcap_{n=0}^{\infty} U_{n}$. Since $1>(M-1) /(M-c) \geq 0$, we can choose $0<s<1$ such that $(M-1)-s(M-c)<0$.

Choose a sequence $\left\{\epsilon_{n}\right\}_{n=0}^{\infty}$ of positive numbers, decreasing to 0 , satisfying $\epsilon_{n-1}(1-$ $\left.s^{n}\right)+s^{n}(M-1-s(M-c))<0, n \geq 1$.

Claim. There exists a decreasing sequence of open sets $\left\{V_{j}\right\}_{j=0}^{\infty}$ such that $V_{0}=X$, $E \cup W=\bigcap_{j=0}^{\infty} V_{j}$ and a sequence of functions $\left\{h_{j}\right\}_{j=0}^{\infty} \subseteq B$ satisfying:

(1) $\operatorname{Re} h_{j}(x)<\operatorname{Re} h_{j}\left(x_{0}\right)=1, x \in E \backslash W, j \geq 0$;

(2) $\left\|h_{j}\right\| \leq M$;

(3) $\operatorname{Re} h_{j}(x)<c, x \notin V_{j}, j \geq 1$; and

(4) $\operatorname{Re} h_{i}(x)<1+\epsilon_{j-1} ; x \in V_{j} \backslash W, i=0,1,2, \ldots, j-1, j \geq 1$. 
Take $V_{0}=X$ and let $h_{0}$ correspond to $V_{0}$. That is, choose $h_{0} \in B$ satisfying (i)-(iv) above with $U=V_{0}$.

Define

$$
Y_{1}=\left\{x \in U_{1} \cap V_{0}: \operatorname{Re} h_{0}(x)<1+\epsilon_{0}\right\} \quad \text { and } \quad V_{1}=Y_{1} \cup W .
$$

Let $h_{1}$ correspond to $V_{1}$. Then $V_{1} \subseteq V_{0}$, (1)-(3) follow from the properties of $h_{1}$ and (4) follows from the definition of $Y_{1}$.

Inductively, assume $V_{0}, \ldots, V_{j}$ and $h_{0}, \ldots, h_{j}$ have been chosen. Define

$$
Y_{j+1}=\left\{x \in U_{j+1} \cap V_{j}: \operatorname{Re} h_{i}(x)<1+\epsilon_{j}, i=0,1,2, \ldots, j\right\}
$$

and

$$
V_{j+1}=Y_{j+1} \cup W
$$

Let $h_{j+1}$ correspond to $V_{j+1}$. Since $Y_{j+1} \subseteq V_{j}$, we have $V_{j+1} \subseteq V_{j}$. By the choice of $h_{j+1}$ it satisfies properties (1)-(3) and the definition of $Y_{j+1}$ yields property (4). Since $E \cup W \subseteq U_{j} \cup W$ for all $j$ and $\bigcap_{j=0}^{\infty} U_{j}=E$, we have $E \cup W=\bigcap_{j=0}^{\infty} V_{j}$. This establishes our claim.

To complete the proof of Theorem 2.5 , let

$$
G=(1-s) \sum_{j=0}^{\infty} s^{j} h_{j} .
$$

Then $G \in B$ and $G\left(x_{0}\right)=1$. If $x \in E \backslash W$, then $\operatorname{Re} G(x)<1$ since $h_{j}(x)<1$ for every $j$. If $x \notin E$, then there exists a maximal $l \geq 0$ such that $x \in V_{l} \backslash V_{l+1}$.

If $l=0$, then $x \in V_{0}$ but $x \notin V_{j}$ for all $j \geq 1$, and

$$
\begin{aligned}
\operatorname{Re} G(x) & =(1-s)\left[\operatorname{Re} h_{0}(x)+\sum_{j=1}^{\infty} s^{j} \operatorname{Re} h_{j}(x)\right] \\
& <(1-s)\left[M+\sum_{j=1}^{\infty} s^{j} c\right]=M+s(c-M)<1 \quad \text { by the choice of } s .
\end{aligned}
$$

If $l \geq 1$,

$$
\begin{aligned}
\operatorname{Re} G(x) & =(1-s)\left[\sum_{j=0}^{l-1} s^{j} \operatorname{Re} h_{j}(x)+s^{l} \operatorname{Re} h_{l}(x)+\sum_{j=l+1}^{\infty} s^{j} \operatorname{Re} h_{j}(x)\right] \\
& <(1-s)\left[\left(1+\epsilon_{l-1}\right) \frac{1-s^{l}}{1-s}+M \cdot s^{l}+c \cdot \frac{s^{l+1}}{1-s}\right] \\
& =1+\epsilon_{l-1}\left(1-s^{l}\right)+s^{l}[M-1-s(M-c)]<1 .
\end{aligned}
$$

Hence

$\operatorname{Re} G(x)<1 \quad$ if $x \notin W, \quad \operatorname{Re} G\left(x_{0}\right)=1$ and $\left\{x \in X: \operatorname{Re} G(x)=\max _{x^{\prime} \in X} \operatorname{Re} G\left(x^{\prime}\right)\right\} \subseteq W \quad$ as claimed. 
Remark 2.6. If, in Theorem 2.5, each $H_{n}$ satisfies

$$
1=\operatorname{Re} H_{n}\left(x_{0}\right)>\operatorname{Re} H_{n}(x), \quad x \in E, x \neq x_{0},
$$

then there is no need for the relatively open neighborhood $W$ of $x_{0}$ in $E$ and one can conclude that there exists $G \in B$ such that $\operatorname{Re} G$ peaks at $x_{0}$.

\section{Proof of the main theorem}

The proof of the main theorem will follow from Lemmas 3.2-3.4 below and Theorem 2.5. For Lemma 3.2 we will need some geometric information about the curve $C_{p}=\left\{(1-x)^{p}:|x|=1\right\}$. For these calculations parametrize $C_{p}$ with $x=-e^{i \varphi}$, $-\pi<\varphi \leq \pi$. We refer to the part of $C_{p}$ where $0<\varphi<\pi$ as the upper branch of $C_{p}$ and the part where $-\pi<\varphi<0$ as the lower branch. Double angle formulas yield

$$
\left(1+e^{i \varphi}\right)^{p}=2^{p}[\cos (\varphi / 2)]^{p} e^{i p \varphi / 2} .
$$

The tangent vector is given by

$$
p\left(1+e^{i \varphi}\right)^{p-1} i e^{i \varphi}=p 2^{p-1}[\cos (\varphi / 2)]^{p-1} i e^{i(p+1) \varphi / 2} .
$$

If $\eta$ is equal to the argument of the tangent vector, then

$$
\eta=\eta(\varphi)=\varphi(p+1) / 2+\pi / 2
$$

$\eta(\varphi)$ is strictly increasing on the interval $(-\pi, \pi)$ and $C_{p}$ is locally convex there. Since

$$
\left|\left(1+e^{i \varphi}\right)^{p}\right|=2^{p}[\cos (\varphi / 2)]^{p},
$$

starting at $2^{p}(\varphi=0)$, the two symmetric branches spiral in different directions around and down to the origin with decreasing modulus. With this parametrization the points where $|\varphi|=2 \pi /(p+1)$ are the turning points introduced in Section 1.

Claim 3.1. Fix $\varphi_{0}$ with $2 \pi /(p+1)<\left|\varphi_{0}\right|<\min (\pi, 2 \pi / p)$. Then the tangent line at $\left(1+e^{i \varphi_{0}}\right)^{p}$ intersects $C_{p}$ at exactly two points, excluding the point of tangency. If $\varphi_{0}>0$, then both of these points lie on the lower branch and, if $\varphi_{0}<0$, then both lie on the upper branch.

We sketch the proof and, due to symmetry, we only consider $\varphi_{0}>0$. The argument of the tangent vector at $\left(1+e^{i \varphi_{0}}\right)^{p}$ satisfies $3 \pi / 2<\eta\left(\varphi_{0}\right)<\min (\pi+p \pi / 2,3 \pi / 2+$ $\pi / p)<2 \pi$. First fix $1<p \leq 2$. Then $2 \pi /(p+1)<\varphi_{0}<\pi$, the curve $C_{p}$ is a simple closed curve and, geometrically, is essentially the same as the cardioid $C_{2}$. The restricted location of the point $\left(1+e^{i \varphi_{0}}\right)^{p}$ on the upper branch between the turning point and the origin, the symmetry, the local convexity of $C_{p}$ and the inclination of the tangent line, as determined by the argument of the tangent vector, yield the result, just as with $C_{2}$. 
Now fix $p>2$. The point where $|\varphi|=2 \pi / p$ is common to both branches and is the point where both cross the negative real axis for the first time. Consequently

$$
C_{p}^{*}=\left\{\left(1+e^{i \varphi}\right)^{p}:-2 \pi / p \leq \varphi \leq 2 \pi / p\right\}
$$

is a simple closed curve and is, geometrically, essentially the same as the cardioid $C_{2}$. As in the case $1<p \leq 2$, the location of the point $\left(1+e^{i \varphi_{0}}\right)^{p}$ on $C_{p}^{*}$ and the geometric properties of $C_{p}^{*}$ yield that the tangent line, excluding the point of tangency, intersects $C_{p}^{*}$ in exactly two points, both on the lower branch.

The local convexity and symmetry of $C_{p}$ show that the tangent line can possibly have additional intersection points on $C_{p}$ only after both branches, starting at $2^{p}$, have made a complete revolution about the origin, that is, when $|\varphi|=4 \pi / p$. If $p<4$, both branches terminate at the origin before there is a complete revolution about the origin, so there are exactly two intersection points as claimed. Thus we can assume $p \geq 4$ and we consider points $\left(1+e^{i \varphi}\right)^{p}$ where $4 \pi / p \leq|\varphi| \leq \pi$. The tangent line at $\left(1+e^{i \varphi_{0}}\right)^{p}$ can be described by $\left(1+e^{i \varphi_{0}}\right)^{p}\left(1+R i e^{i \varphi_{0} / 2}\right),-\infty<R<\infty$. Then a straightforward calculation shows that $2^{p}\left[\cos \left(\varphi_{0} / 2\right)\right]^{p+1}$ is the minimum modulus of points on the tangent line at $\left(1+e^{i \varphi_{0}}\right)^{p}$. Then, for $4 \pi / p \leq|\varphi| \leq \pi$,

$$
\begin{aligned}
\left|\left(1+e^{i \varphi}\right)^{p}\right| & =2^{p}[\cos (\varphi / 2)]^{p} \leq 2^{p}[\cos (2 \pi / p)]^{p}=2^{p}\left[2(\cos (\pi / p))^{2}-1\right]^{p} \\
& \leq 2^{p}[\cos (\pi / p)]^{2 p} \\
& <2^{p}[\cos (\pi / p)]^{p+1} \\
& <2^{p}\left[\cos \left(\varphi_{0} / 2\right)\right]^{p+1} .
\end{aligned}
$$

Hence the tangent line avoids this part of $C_{p}$ and the claim follows for all $p>1$.

Our eventual goal is to apply Theorem 2.5 and, to this end, we need to construct appropriate functions $H$ and $H_{n}$.

Lemma 3.2. For a given $p>1$ and $q>0$, there exist $x_{0}=\mathrm{e}^{i \theta_{0}}$, where $0<\left|\theta_{0}\right|<$ $\pi(p-1) /(p+1)$, and $C \in \mathbb{C}$ such that

$$
H(x, y)= \begin{cases}-\bar{x}_{0}(q-p x) y & \text { on } \Gamma \times \Gamma \backslash\{1\} \\ -\bar{x}_{0}(q-p x)-C(1-x)^{p} & \text { on } \Gamma \times\{1\}\end{cases}
$$

satisfies:

(1) $\sup \operatorname{Re} H(x, y)<\operatorname{Re} H\left(x_{0}, 1\right), y \neq 1$; and

(2) $\operatorname{Re} H(x, 1)<\operatorname{Re} H\left(x_{0}, 1\right), x \neq x_{0}$.

Proof. First observe that, if we can find $x_{0}$ and $C$ that satisfy the lemma, then by symmetry $\bar{x}_{0}$ and $\bar{C}$ will also satisfy the lemma with an appropriately modified $H$. Thus, it suffices to assume that $\theta_{0}<0$. Let

$$
\theta^{*}=-\pi(p-1) /(p+1) \text { and } \theta_{0}=\theta^{*}+\epsilon, \epsilon>0 .
$$




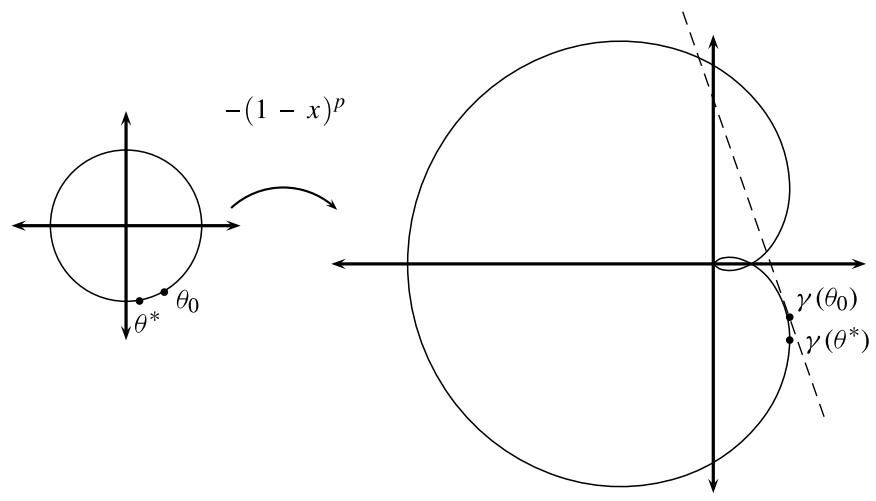

FIGURE 1. Illustration of Lemma 3.2 for the curve $\gamma=-C_{p}$.

Reflect the curve $C_{p}$ through the origin and parametrize $\gamma=-C_{p}=\left\{-(1-x)^{p}:|x|=1\right\}$ with $x=e^{i \theta}$ and

$$
\gamma(\theta)=-\left(1-e^{i \theta}\right)^{p}=e^{-i \pi}\left(1+e^{i(\theta+\pi)}\right)^{p}, \quad-\pi<\theta \leq \pi .
$$

Then $\gamma\left(\theta^{*}\right)$ is a reflected turning point in the lower half plane. With this parametrization of $\gamma$, the tangent line at $\gamma\left(\theta_{0}\right)$ satisfies Claim 3.1 for small $\epsilon>0$ and the two intersection points lie on the part of $\gamma$ in the upper half plane (see Figure 1).

Recall that $\eta(\varphi)$, the argument of the tangent vector for the curve $\left(1+e^{i \varphi}\right)^{p}$, is given by $\eta(\varphi)=\varphi(p+1) / 2+\pi / 2$ so that the argument of the tangent vector at the point $\gamma\left(\theta_{0}\right)$ is given by $\epsilon(p+1) / 2+\pi / 2$. Let $\alpha=\alpha\left(\theta_{0}\right)=-\epsilon(p+1) / 2$. Then the rotated curve $\gamma_{r}(\theta)=e^{i \alpha} \gamma(\theta)$ has a vertical tangent at $\gamma_{r}\left(\theta_{0}\right)$. Define $T(\theta)=T\left(\theta_{0}, \theta\right)=\operatorname{Re} \gamma_{r}(\theta)$. By the choice of $\alpha$, the local convexity of $\gamma$ and Claim 3.1, T( $\theta)$ achieves a local maximum at $\theta_{0}, T(\theta)$ achieves a unique maximum for some value of $\theta$, say $\theta=\beta$, and there exist two distinguished angles $\varphi_{1}=\varphi_{1}\left(\theta_{0}\right), \varphi_{2}=\varphi_{2}\left(\theta_{0}\right), \varphi_{1}, \varphi_{2}>0$, such that $T\left(\beta-\varphi_{1}\right)=$ $T\left(\beta+\varphi_{2}\right)=T\left(\theta_{0}\right)$. Also $T(\theta)<T\left(\theta_{0}\right)$ if $\theta \notin\left[\beta-\varphi_{1}, \beta+\varphi_{2}\right]$ (see Figure 2).

Define $g(\theta)=g\left(\theta_{0}, \theta\right)=\operatorname{Re}\left[-\bar{x}_{0}(q-p x)\right]$ so that $g(\theta)$ has a unique maximum at $\theta_{0}$. Another straightforward calculation yields that

$$
T\left(\theta_{0}, \theta_{0}\right)=2^{p}\{\cos [\epsilon / 2+\pi /(p+1)]\}^{p+1} .
$$

Then, as $\epsilon$ decreases to 0 :

(i) $1+\cos \left(\theta_{0}\right)$ decreases to $1+\cos \left(\theta^{*}\right)$;

(ii) $\alpha=\alpha\left(\theta_{0}\right)$ increases to 0 ;

(iii) $T\left(\theta_{0}\right)=T\left(\theta_{0}, \theta_{0}\right)$ increases to $T\left(\theta^{*}, \theta^{*}\right)=T\left(\theta^{*},-\theta^{*}\right)$;

(iv) $g\left(\theta_{0}\right)=g\left(\theta_{0}, \theta_{0}\right)$ converges to $g\left(\theta^{*}, \theta^{*}\right)>g\left(\theta^{*},-\theta^{*}\right)$;

(v) $\beta$ converges to $-\theta^{*}$;

(vi) the interval $\left[\beta-\varphi_{1}, \beta+\varphi_{2}\right]$ decreases to the point $\left\{-\theta^{*}\right\}$.

Let $R^{*}=q\left(1+\cos \left(\theta^{*}\right)\right) / T\left(\theta^{*}, \theta^{*}\right)$ and fix $R$ with $R^{*}<R<2 R^{*}$. Let $\sigma=g\left(\theta^{*}, \theta^{*}\right)-$ $g\left(\theta^{*},-\theta^{*}\right)>0$. Then we can chose $\epsilon$ sufficiently close to 0 so that: 


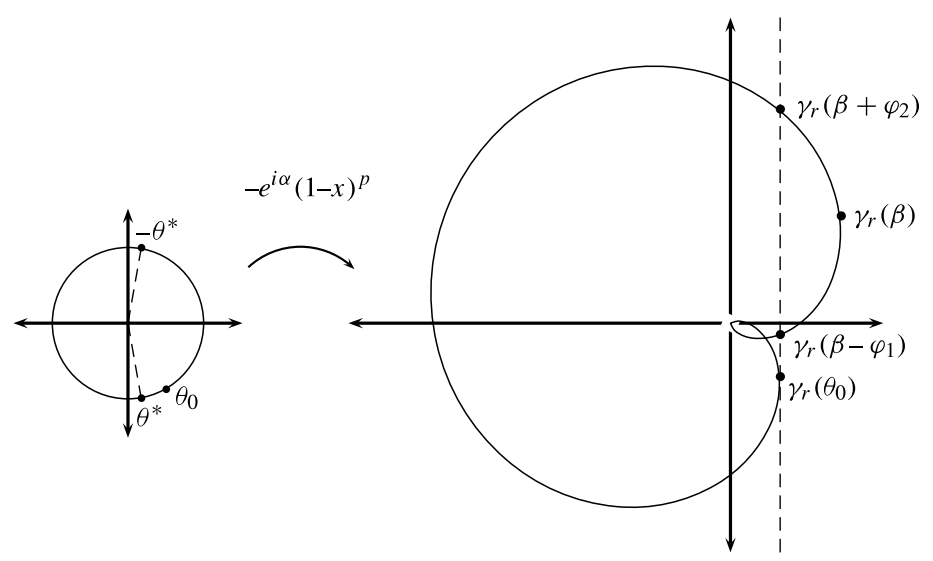

FIGURE 2. Illustration of the curve $\gamma_{r}(\theta)$.

(a) $g\left(\theta_{0}\right)-\sup \left\{g(\theta): \theta \in\left[\beta-\varphi_{1}, \beta+\varphi_{2}\right]\right\}>\sigma / 2$ by (iv)-(vi);

(b) $\left|T(\beta)-T\left(\theta_{0}\right)\right|<\sigma /\left(8 R^{*}\right)$ by (iii) and (v);

(c) $|T(\theta)-T(\beta)|<\sigma /\left(8 R^{*}\right), \theta \in\left[\beta-\varphi_{1}, \beta+\varphi_{2}\right]$ by (v) and (vi);

(d) $\quad R>q\left(1+\cos \left(\theta_{0}\right)\right) / T\left(\theta_{0}, \theta_{0}\right)>R^{*}$ by (i) and (iii).

So fix $\theta_{0}$ satisfying (a)-(d), fix $\alpha=\alpha\left(\theta_{0}\right)$, let $C=\operatorname{Re}^{i \alpha}$ and let $f(\theta)=R T\left(\theta_{0}, \theta\right)=$ $R T(\theta)$. Then $\operatorname{Re} H(x, 1)=g(\theta)+f(\theta)$. We claim that (1) and (2) in the lemma obtain with this choice of $\theta_{0}$ and $C$.

Since $\left|\bar{x}_{0}(q-p x) y\right| \leq q+p$, to achieve (1) we want $q+p<f\left(\theta_{0}\right)+g\left(\theta_{0}\right)=$ $R \cdot T\left(\theta_{0}\right)-q \cos \left(\theta_{0}\right)+p$, which, since $T\left(\theta_{0}, \theta_{0}\right)>0$, is equivalent to $R>q(1+$ $\left.\cos \left(\theta_{0}\right)\right) / T\left(\theta_{0}, \theta_{0}\right)$, which is $(\mathrm{d})$.

Next we want $f\left(\theta_{0}\right)+g\left(\theta_{0}\right)>f(\theta)+g(\theta)$ for $-\pi<\theta \leq \pi$ and $\theta \neq \theta_{0}$. Since $g(\theta)$ peaks at $\theta_{0}$, for $\theta \notin\left[\beta-\varphi_{1}, \beta+\varphi_{2}\right]$ we have $T(\theta)<T\left(\theta_{0}\right)$ and, hence, $f\left(\theta_{0}\right)+g\left(\theta_{0}\right)>$ $f(\theta)+g(\theta)$. If $\theta \in\left[\beta-\varphi_{1}, \beta+\varphi_{2}\right]$, then by (a) we have $g\left(\theta_{0}\right)>\sigma / 2+g(\theta)$. Also by (b) and (c) we have $\left|T(\theta)-T\left(\theta_{0}\right)\right|<\sigma /\left(4 R^{*}\right)$ so that $\left|f(\theta)-f\left(\theta_{0}\right)\right|<\sigma R /\left(4 R^{*}\right)<\sigma / 2$. Hence

$$
f\left(\theta_{0}\right)+g\left(\theta_{0}\right)>(f(\theta)-\sigma / 2)+(\sigma / 2+g(\theta))=f(\theta)+g(\theta),
$$

which establishes Lemma 3.2.

LeMma 3.3. Let $\left\{f_{n}\right\}$ be a sequence of continuous functions that converges to $f(x)=(1-x)^{p}$ uniformly on $\Gamma$. Let $(1+y)^{n} / 2^{n}=\sum_{i=0}^{n} b_{n_{i}} y^{i}$ on $\Gamma$ and $g_{n}(x, y)=$ $y^{n} \sum_{i=0}^{n} b_{n_{i}} f_{n+i}(x) y^{i}$ on $\mathbb{T}$. Then $g_{n}(x, y)$ converges to 0 uniformly on compact subsets of $\Gamma \times \Gamma \backslash\{1\}$ and uniformly to $(1-x)^{p}$ on $\Gamma \times\{1\}$. 
Proof. We have $b_{n_{i}}>0$ and $\sum_{i=0}^{n} b_{n_{i}}=1$. Rewrite

$$
\begin{aligned}
g_{n}(x, y) & =y^{n} \sum_{i=0}^{n} b_{n_{i}} y^{i} f_{n}(x)+y^{n} \sum_{i=0}^{n} b_{n_{i}} y^{i}\left(f_{n+i}(x)-f_{n}(x)\right) \\
& =y^{n}\left(\frac{1+y}{2}\right)^{n} f_{n}(x)+y^{n} \sum_{i=0}^{n} b_{n_{i}} y^{i}\left(f_{n+i}(x)-f_{n}(x)\right) .
\end{aligned}
$$

Let $\epsilon>0$. Since $\left\{f_{n}\right\}$ is uniformly Cauchy on $\Gamma,\left|f_{n+i}-f_{n}\right|<\epsilon$ on $\Gamma$ for large $n$. Therefore

$$
\left|y^{n} \sum_{i=o}^{n} b_{n_{i}} y^{i}\left(f_{n+i}(x)-f_{n}(x)\right)\right|<\sum_{i=o}^{n} b_{n_{i}} \epsilon=\epsilon \quad \text { on } \mathbb{T} \text { for large } n .
$$

Thus the second term in (3.1) converges to 0 uniformly on $\mathbb{T}$. By hypothesis, $f_{n}(x)$ converges to $f(x)=(1-x)^{p}$ uniformly on $\Gamma \times\{1\}$ and, since $(1+y)^{n} / 2^{n}$ converges to 0 uniformly on compact subsets of $\Gamma \backslash\{1\}$, the first term converges to 0 uniformly on compact subsets of $\Gamma \times \Gamma \backslash\{1\}$. Hence $g_{n}(x, y)$ converges as stated in the lemma.

Lemma 3.4. For $p>1$ and $q>0, \quad K_{n}(x, 1) / B_{n}(q)$ converges to $f(x)=(1-x)^{p}$ uniformly on $\Gamma$.

Proof. We have

$$
f(x)=(1-x)^{p}=\sum_{n=0}^{\infty} A_{n}(p) x^{n}
$$

where

$$
A_{0}(p)=1 \quad \text { and } \quad A_{n}(p)=(-1)^{n} \frac{p(p-1) \cdots(p-n+1)}{n !}, \quad n \geq 1 .
$$

Let $p=s+r, 0 \leq r<1, s=1,2,3, \ldots$ Let $M=p(p-1) \cdots(p-s+1)=(s+r)$ $(s+r-1) \cdots(1+r)$. Then, for $n=1,2,3, \ldots, s+1$,

$$
\left|A_{n}(p)\right|=\frac{(s+r)(s+r-1) \cdots(s+r-n+1)}{n !} .
$$

For $n \geq s+2$,

$$
\begin{aligned}
\left|A_{n}(p)\right| & =\frac{M \cdot r(1-r)(2-r) \cdots(n-(s+1)-r)}{n !} \\
& =M \cdot r \cdot\left(\frac{1-r}{1}\right) \cdot\left(\frac{2-r}{2}\right) \cdots\left(\frac{n-(s+1)-r}{n-(s+1)}\right) \cdot \frac{1}{n-s} \cdots \frac{1}{n} .
\end{aligned}
$$

Since $(s+r)-j \geq 1$ for $j=0,1,2, \ldots, s-1$, it follows that, for all $n,\left|A_{n}(p)\right| \leq M$ and, for $n \geq 2,\left|A_{n}(p)\right| \leq M /(n-1) n$.

Let $f(x)=T_{N}(x)+R_{N}(x)$ where

$$
T_{N}(x)=\sum_{i=0}^{N} A_{i}(p) x^{i} \quad \text { and } \quad R_{N}(x)=\sum_{i=N+1}^{\infty} A_{i}(p) x^{i} .
$$


For $n>N$, consider

$$
\begin{aligned}
\left|\frac{K_{n}(x, 1)}{B_{n}(q)}-f(x)\right| & =\left|\sum_{i=0}^{n} A_{i}(p) x^{i} \frac{B_{n-i}(q)}{B_{n}(q)}-T_{N}(x)-R_{N}(x)\right| \\
& \leq\left|\sum_{i=0}^{N} A_{i}(p) x^{i}\left(\frac{B_{n-i}(q)}{B_{n}(q)}-1\right)\right|+\left|\sum_{i=N+1}^{n} A_{i}(p) x^{i} \frac{B_{n-i}(q)}{B_{n}(q)}\right|+\left|R_{N}(x)\right| .
\end{aligned}
$$

Let $\epsilon>0$. Choose $N_{1}$ such that $\sum_{i=N_{1}+1}^{\infty} M /\left(i^{2}-i\right)<\epsilon / 3$. Then $\left|R_{N}(x)\right|<\epsilon / 3$ if $N \geq N_{1}$.

Let $n=i+k$ so that $B_{n-i}(q) / B_{n}(q)=B_{k}(q) / B_{k+i}(q)$. Since $B_{k+1}(q)=B_{k}(q)(k+q) /$ $(k+1)$ and $B_{0}(q)=1, B_{n-i}(q) / B_{n}(q) \leq 1$ for $0 \leq i \leq n, q \geq 1$.

If $0<q<1$, then

$$
\frac{B_{k}(q)}{B_{k+i}(q)}=\frac{(k+1)(k+2) \cdots(k+i)}{(k+q)(k+q+1) \cdots(k+q+i-1)}<\left(\frac{1}{q}\right)\left(\frac{2}{q+1}\right) \cdots\left(\frac{i}{q+i-1}\right) .
$$

Recall that the gamma function satisfies

$$
\Gamma(q)=\lim _{i \rightarrow \infty} \frac{(i-1) !(i-1)^{q}}{q(q+1) \cdots(q+i-1)} .
$$

Choose $N_{2}$ so that, if $i>N_{2}$, then

$$
\frac{(i-1) !(i-1)^{q}}{q(q+1) \cdots(q+i-1)}<2 \Gamma(q)
$$

and hence

$$
\frac{B_{n-i}(q)}{B_{n}(q)}<\frac{i !}{q(q+1) \cdots(q+i-1)}<\frac{2 \Gamma(q) \cdot i}{(i-1)^{q}} .
$$

Choose $N_{3}$ so that, if $m>N_{3}$, then

$$
\sum_{i=m}^{\infty} \frac{1}{(i-1)^{1+q}}<\frac{\epsilon}{6 \Gamma(q) M} .
$$

Fix $N=\max \left\{N_{1}, N_{2}, N_{3}\right\}$. Then $\left|R_{N}(x)\right|<\epsilon / 3$. For $n>N$,

$$
\text { if } q \geq 1, \quad\left|\sum_{i=N+1}^{n} A_{i}(p) x^{i} \frac{B_{n-i}(q)}{B_{n}(q)}\right| \leq \sum_{i=N+1}^{n}\left|A_{i}(p)\right| \leq \sum_{i=N+1}^{\infty} \frac{M}{(i-1) i}<\frac{\epsilon}{3}
$$

and,

$$
\text { if } \begin{aligned}
0<q<1, \quad\left|\sum_{i=N+1}^{n} A_{i}(p) x^{i} \frac{B_{n-i}(q)}{B_{n}(q)}\right| & \leq \sum_{i=N+1}^{n} \frac{M}{(i-1) i} \cdot \frac{2 \Gamma(q) i}{(i-1)^{q}} \\
& =2 M \Gamma(q) \sum_{i=N+1}^{n} \frac{1}{(i-1)^{1+q}}<\frac{\epsilon}{3} .
\end{aligned}
$$


Now observe that, for each fixed $i, B_{k}(q) / B_{k+i}(q)$ converges to 1 as $k \rightarrow \infty$. Hence there exists $N_{0} \geq N$ such that, if $n \geq N_{0}$, then $\left|1-B_{n-i}(q) / B_{n}(q)\right|<\epsilon /(3 N M)$ for each $i, 0 \leq i \leq N$. Consequently

$$
\left|\sum_{i=0}^{N} A_{i}(p) x^{i}\left(1-\frac{B_{n-i}(q)}{B_{n}(q)}\right)\right|<N M \frac{\epsilon}{3 N M}=\frac{\epsilon}{3} \quad \text { if } n \geq N_{0} .
$$

Thus, given $\epsilon>0$, there exists $N_{0}$ such that

$$
\left|\frac{K_{n}(x, 1)}{B_{n}(q)}-f(x)\right|<\epsilon \quad \text { on } \Gamma \text {, if } n \geq N_{0} .
$$

That is, $K_{n}(x, 1) / B_{n}(q)$ converges to $f(x)$ uniformly on $\Gamma$.

Proof (Main theorem). In Theorem 2.5 let $X=\mathbb{T}, E=\Gamma \times\{1\}$ and $B=\overline{S p}\left\{K_{n}: n=\right.$ $0,1,2, \ldots$,$\} . Replace x_{0}=e^{i \theta_{0}}$ with $x_{1}=e^{i \theta_{1}}$ and let $x_{1}, C$ and $H$ be as in Lemma 3.2. Let $f_{n}(x)=K_{n}(x, 1) / B_{n}(q)$ in Lemma 3.3 and choose a relatively open neighborhood $W$ of $\left(x_{1}, 1\right)$ in $\Gamma \times\{1\}$. Finally, let

$$
\begin{aligned}
H_{n}(x, y) & =-\bar{x}_{1} K_{1}(x, y)-C \sum_{i=0}^{n} \frac{b_{n_{i}}}{B_{n+i}(q)} K_{n+i}(x, y) \\
& =-\bar{x}_{1}(q-p x) y-C y^{n} \sum_{i=0}^{n} b_{n_{i}} f_{n+i}(x) y^{i}
\end{aligned}
$$

and normalize $H$ and $H_{n}$ with $\widetilde{H}=H / \operatorname{Re} H\left(x_{1}, 1\right)$ and $\widetilde{H}_{n}=H_{n} / \operatorname{Re}_{n}\left(x_{1}, 1\right)$. We verify that the hypotheses of Theorem 2.5 are satisfied with the functions $\widetilde{H_{n}}$ and $\widetilde{H}$ :

$$
\begin{aligned}
\left|\sum_{i=0}^{n} b_{n_{i}} f_{n+i}(x) y^{i}\right| & \leq\left|\sum_{i=0}^{n} b_{n_{i}}\left(f_{n+i}(x)-f_{n}(x)\right) y^{i}\right|+\left|\sum_{i=0}^{n} b_{n_{i}} y^{i} f_{n}(x)\right| \\
& =\left|\sum_{i=0}^{n} b_{n_{i}}\left(f_{n+i}(x)-f_{n}(x)\right) y^{i}\right|+\left|\left(\frac{1+y}{2}\right)^{n} f_{n}(x)\right| .
\end{aligned}
$$

By Lemma 3.4, $f_{n}$ converges to $(1-x)^{p}$ uniformly on $\Gamma$. It follows that there exists $M \geq 1$ such that $\left\|\widetilde{H}_{n}\right\|<M$ for all $n$. From Lemma $3.3, \widetilde{H}_{n}$ converges to $\widetilde{H}$ uniformly on $\Gamma \times\{1\}$. The normalization yields $\operatorname{Re} \widetilde{H}_{n}\left(x_{1}, 1\right)=\operatorname{Re} \widetilde{H}\left(x_{1}, 1\right)=1$. From Lemma 3.2, Re $\widetilde{H}(x, 1)<\operatorname{Re} \widetilde{H}\left(x_{1}, 1\right), x \neq x_{1}$, and there exists $c$ such that sup $\operatorname{Re} \widetilde{H}(x, y)<c<$ $1=\operatorname{Re} \widetilde{H}\left(x_{1}, 1\right)$ on $\Gamma \times \Gamma \backslash\{1\}$. From Lemma $3.3, \widetilde{H}_{n}$ converges to $\widetilde{H}$ uniformly on compact subsets of $\Gamma \times \Gamma \backslash\{1\}$. Thus all of the hypotheses of Theorem 2.5 are fulfilled and there exists $G \in B$ such that

$$
K=\left\{(x, y) \in \mathbb{T}: \operatorname{Re} G(x, y)=\max _{\left(x^{\prime}, y^{\prime}\right) \in \mathbb{T}} \operatorname{Re} G\left(x^{\prime}, y^{\prime}\right)\right\} \subseteq W .
$$


If one considers the generalized functional $L_{G}$ corresponding to $G$ and the set of generalized support points

$$
\Sigma=\left\{k_{x, y}: \operatorname{Re} L_{G}\left(k_{x, y}\right)=\max _{k_{x^{\prime}, y^{\prime}} \in F} \operatorname{Re} L_{G}\left(k_{x^{\prime}, y^{\prime}}\right)\right\},
$$

then $f_{\mu} \in \Sigma$ if and only if the closed support of $\mu$ is contained in $K$. According to our Remark 2.1, there exist extreme points in $\Sigma$ and, hence, pairs $\left(x_{0}, 1\right) \in W$ such that $k_{x_{0}, 1}$ is an extreme point of $F$. Moreover, since $W$ is an arbitrary relatively open neighborhood of $\left(x_{1}, 1\right), x_{1}=e^{i \theta_{1}}$, and $\left|\theta_{1}\right|<\pi(p-1) /(p+1)$, there exist extreme points $k_{x_{0}, 1}, x_{0}=e^{i \theta_{0}}$, with $x_{0}$ arbitrarily close to $x_{1}$ and satisfying $\left|\theta_{0}\right|<\pi(p-1) /(p+1)$ as claimed.

Corollary 3.5. For $p>1$ and $q>0$ there exist $\delta>0$ and a dense subset $J_{e}$ of $J=$ $\left\{x=e^{i \theta}: \pi(p-1) /(p+1)-\delta<|\theta|<\pi(p-1) /(p+1)\right\}$ such that $k_{x, 1}$ is an extreme point of $F$ for each $x \in J_{e}$.

Proof. By the symmetry of the curve $(1-x)^{p}$, the arguments in the proof of Lemma 3.2 demonstrate that:

(i) if $x_{0}=e^{i \theta_{0}}$ satisfies Lemma 3.2, then so does $\bar{x}_{0}=e^{-i \theta_{0}}$;

(ii) if $\left(1-x_{0}\right)^{p}$ is sufficiently close to the turning point $\left(1-x^{*}\right)^{p}$, then $x_{0}$ and $\bar{x}_{0}$ both satisfy Lemma 3.2.

Therefore we can choose $\delta$ so that, if $x_{0} \in J$ as defined above, then $x_{0}$ satisfies Lemma 3.2. The proof of the main theorem then shows that any relatively open neighborhood of a point in $J$ contains a point $x$ such that $k_{x, 1}$ is an extreme point of $F$. Take $J_{e}=\left\{x \in J: k_{x, 1}\right.$ is an extreme point of $\left.F\right\}$.

Remark 3.6. According to Remarks 2.1 and 2.6, if the functions $H_{n}$ in Theorem 2.5 actually peak on $E$, then there is no need to consider relatively open neighborhoods and one could conclude that there is an interval beyond each turning point that yields extreme points of $F$. This is actually the case when $p$ is an integer, $p=m \geq 2$.

Theorem 3.7. Fix $q>0$ and $p=m(m=2,3,4, \ldots)$. Then there exist functions $H_{n} \in$ $\overline{S p}\left\{K_{n}: n=0,1,2, \ldots,\right\}$ such that:

(i) $H_{n}(x, 1)=H(x, 1)=-\bar{x}_{0}(q-m x)-C(1-x)^{m}$;

(ii) $H_{n}(x, y)$ converges to $H(x, y)=-\bar{x}_{0}(q-m x) y$ uniformly on compact subsets of $\Gamma \times \Gamma \backslash\{1\}$.

(See the function $H(x, y)$ in Lemma 3.2.)

We will need the following lemma in the proof of Theorem 3.7.

Lemma 3.8. Fix $q>0$ and $p=m$. Let $n^{*}=\min (n, m)$. Then

$$
K_{n}(x, 1)=\sum_{l=0}^{n^{*}}\left(\begin{array}{c}
m \\
l
\end{array}\right) B_{n-l}(q-m+l)(1-x)^{l} .
$$


Proof. We have

$$
\frac{(1-x z)^{m}}{(1-z)^{q}}=\sum_{n=1}^{\infty} K_{n}(x, 1) z^{n}
$$

Let $t=1-x, x=1-t$.

$$
(1-x z)^{m}=(1-(1-t) z)^{m}=(1-z+t z)^{m}=\sum_{l=0}^{m}\left(\begin{array}{c}
m \\
l
\end{array}\right) t^{l} z^{l}(1-z)^{m-l} .
$$

So

$$
\begin{aligned}
\frac{(1-x z)^{m}}{(1-z)^{q}} & =\sum_{l=0}^{m}\left(\begin{array}{c}
m \\
l
\end{array}\right) t^{l} z^{l}(1-z)^{m-l-q} \\
& =\sum_{l=0}^{m}\left(\begin{array}{c}
m \\
l
\end{array}\right) t^{l} z^{l} \sum_{k=0}^{\infty} A_{k}(m-l-q) z^{k} \\
& =\sum_{l=0}^{m}\left(\begin{array}{c}
m \\
l
\end{array}\right) t^{l} \sum_{k=0}^{\infty} A_{k}(m-l-q) z^{k+l} .
\end{aligned}
$$

When $m-l-q$ is a nonnegative integer, this series is a finite sum.

Let $n=k+l, k=n-l$. Then

$$
\begin{aligned}
\frac{(1-x z)^{m}}{(1-z)^{q}} & =\sum_{l=0}^{m}\left(\begin{array}{c}
m \\
l
\end{array}\right) t^{l} \sum_{n=l}^{\infty} A_{n-l}(m-l-q) z^{n} \\
& =\sum_{n=0}^{\infty}\left[\sum_{l=0}^{n^{*}}\left(\begin{array}{c}
m \\
l
\end{array}\right) A_{n-l}(m-l-q) t^{l}\right] z^{n} .
\end{aligned}
$$

Since $A_{n-l}(m-l-q)=B_{n-l}(q-m+l)$,

$$
K_{n}(x, 1)=\sum_{l=0}^{n^{*}}\left(\begin{array}{c}
m \\
l
\end{array}\right) B_{n-l}(q-m+l)(1-x)^{l} .
$$

Proof of Theorem 3.7. For fixed $n \geq m$, let

$$
H_{n}(x, y)=\sum_{j=0}^{m-1} D_{j}(n) K_{j}(x, y)-C \sum_{i=0}^{n} \frac{b_{n_{i}}}{B_{n+i-m}(q)} K_{n+i}(x, y),
$$

where $b_{n_{i}}$ is as in Lemma 3.3 and where $D_{0}(n), D_{1}(n), \ldots, D_{m-1}(n)$ are to be determined. Since $K_{j}(x, y)=K_{j}(x, 1) y^{j}$ for all $j=0,1,2, \ldots$, by Lemma 3.8,

$$
\begin{aligned}
H_{n}(x, y)= & \sum_{j=0}^{m-1} D_{j}(n)\left[\sum_{l=0}^{j}\left(\begin{array}{c}
m \\
l
\end{array}\right) B_{j-l}(q-m+l)(1-x)^{l}\right] y^{j} \\
& -C \sum_{i=0}^{n} \frac{b_{n_{i}}}{B_{n+i-m}(q)}\left[\sum_{l=0}^{m}\left(\begin{array}{c}
m \\
l
\end{array}\right) B_{n+i-l}(q-m+l)(1-x)^{l}\right] y^{n+i} .
\end{aligned}
$$


Let

$$
\alpha_{j, l}=\left(\begin{array}{c}
m \\
l
\end{array}\right) B_{j-l}(q-m+l), \quad j=0,1,2, \ldots, m-1, l=0,1,2, \ldots, j,
$$

and

$$
\beta_{n, l}(y)=\left(\begin{array}{c}
m \\
l
\end{array}\right) \sum_{i=0}^{n} \frac{b_{n_{i}} B_{n+i-l}(q-m+l)}{B_{n+i-m}(q)} y^{i} .
$$

Then

$$
\begin{aligned}
H_{n}(x, y)= & \sum_{j=0}^{m-1} D_{j}(n)\left[\sum_{l=0}^{j} \alpha_{j, l}(1-x)^{l}\right] y^{j} \\
& \quad-C y^{n} \sum_{l=0}^{m-1} \beta_{n, l}(y)(1-x)^{l}-C y^{n}(1-x)^{m}\left(\frac{1+y}{2}\right)^{n}
\end{aligned}
$$

and

$$
H_{n}(x, 1)=\sum_{j=0}^{m-1} D_{j}(n)\left[\sum_{l=0}^{j} \alpha_{j, l}(1-x)^{l}\right]-C \sum_{l=0}^{m-1} \beta_{n, l}(1)(1-x)^{l}-C(1-x)^{m}
$$

To achieve (i) in Theorem 3.7, we want to choose $D_{0}(n), \ldots, D_{m-1}(n)$ so that

$$
H_{n}(x, 1)=-\bar{x}_{0}(q-m)-\bar{x}_{0} m(1-x)-C(1-x)^{m} .
$$

This generates the following system of equations to be satisfied:

$$
\begin{aligned}
& \sum_{j=0}^{m-1} D_{j}(n) \alpha_{j, 0}=-\bar{x}_{0}(q-m)+C \beta_{n, 0}(1) . \\
& \sum_{j=1}^{m-1} D_{j}(n) \alpha_{j, 1}=-\bar{x}_{0} m+C \beta_{n, 1}(1) . \\
& \sum_{j=l}^{m-1} D_{j}(n) \alpha_{j, l}=C \beta_{n, l}(1), \quad l=2,3, \ldots, m-1 .
\end{aligned}
$$

Note that the coefficient matrix associated with the variables $D_{0}(n), D_{1}(n), \ldots, D_{m-1}$ $(n)$ is a triangular matrix with entries down the main diagonal given by

$$
\alpha_{l, l}=\left(\begin{array}{c}
m \\
l
\end{array}\right) B_{0}(q-m+l)=\left(\begin{array}{c}
m \\
l
\end{array}\right), \quad l=0,1, \ldots, m-1 .
$$

Hence this system of equations has unique solutions $D_{j}(n), j=0,1,2, \ldots, m-1$. With these choices in the definition of $H_{n}$, we obtain (i) in Theorem 3.7. It remains to show that $H_{n}(x, y)$ converges to $-\bar{x}_{0}(q-m x) y$ uniformly on compact subsets 
of $\Gamma \times \Gamma \backslash\{1\}$. We first note that $(1+y)^{n} / 2^{n}$ converges to 0 uniformly on compact subsets of $\Gamma \times\{1\}$. Also,

$$
\begin{aligned}
\beta_{n, l}(y) & =\left(\begin{array}{c}
m \\
l
\end{array}\right)\left[\sum_{i=0}^{n} \frac{b_{n_{i}} B_{n+i-l}(q-m+l)}{B_{n+i-m}(q)} y^{i}\right] \\
& =\left(\begin{array}{c}
m \\
l
\end{array}\right)\left[\sum_{i=0}^{n} \frac{b_{n_{i}}(q-m+l)(q-m+l+1) \cdots(q-1)}{(n+i-l)(n+i-l-1) \cdots(n+i-m+1)} y^{i}\right] \\
& =\left(\begin{array}{c}
m \\
l
\end{array}\right) r(m, q, l)\left[\sum_{i=0}^{n} \frac{b_{n_{i}}}{(n+i-l)(n+i-l-1) \cdots(n+i-m+1)} y^{i}\right],
\end{aligned}
$$

where $r(m, q, l)=(q-m+l)(q-m+l+1) \cdots(q-1)$. Then

$$
\begin{aligned}
\left|\beta_{n, l}(y)\right| & \leq\left(\begin{array}{c}
m \\
l
\end{array}\right)|r(m, q, l)| \sum_{i=0}^{n} \frac{b_{n_{i}}}{(n+i-l)(n+i-l-1) \cdots(n+i-m+1)} \\
& \leq\left(\begin{array}{c}
m \\
l
\end{array}\right)|r(m, q, l)| \frac{1}{n-m+1} \sum_{i=0}^{n} b_{n_{i}} \\
& =\frac{\left(\begin{array}{c}
m \\
l
\end{array}\right)|r(m, q, l)|}{n-m+1} .
\end{aligned}
$$

Therefore $\beta_{n, l}(y)$ converges to 0 uniformly on $|y|=1$ for $l=0,1,2, \ldots, m-1$. If we let $n \rightarrow \infty$ in our system of equations, then $D_{j}=\lim _{n \rightarrow \infty} D_{j}(n)$ exists for each $j=0,1,2, \ldots, m-1$ and

$$
\begin{aligned}
& \sum_{j=0}^{m-1} D_{j} \alpha_{j, 0}=-\bar{x}_{0}(q-m), \\
& \sum_{j=1}^{m-1} D_{j} \alpha_{j, 1}=-\bar{x}_{0} m
\end{aligned}
$$

and

$$
\sum_{j=l}^{m-1} D_{j} \alpha_{j, l}=0, \quad l=2,3, \ldots, m-1
$$

Since

$$
\begin{aligned}
& \alpha_{1,1}=\left(\begin{array}{c}
m \\
1
\end{array}\right) B_{0}(q-m+1)=m, \\
& \alpha_{0,0}=\left(\begin{array}{c}
m \\
0
\end{array}\right) B_{0}(q-m)=1, \\
& \alpha_{1,0}=\left(\begin{array}{c}
m \\
0
\end{array}\right) B_{1}(q-m)=q-m,
\end{aligned}
$$


solving these equations yields $D_{j}=0, j=2,3, \ldots, m-1, D_{1}=-\bar{x}_{0}$ and $D_{0}=0$. Therefore the first sum of $H_{n}(x, y)$ converges uniformly to $-\bar{x}_{0}(q-m x) y$ and the second sum converges to 0 uniformly on compact subsets of $\Gamma \times \Gamma \backslash\{1\}$. This establishes (ii) in Theorem 3.7.

Corollary 3.9. For fixed $q>0$ and $p=m(m=2,3, \ldots)$, there exists $\delta>0$ such that, if

$$
x \in J=\left\{\mathrm{e}^{i \theta}: \pi(m-1) /(m+1)-\delta<|\theta|<\pi(m-1) /(m+1)\right\},
$$

then $k_{x, 1}$ is an extreme point of $F$.

Proof. Theorem 3.7, the proof of Lemma 3.2 and Remark 3.6 yield the result.

It is almost certainly the case that, for any $p>1$ and $q>0$, there is an interval $J$, as in Corollary 3.9, with $k_{x, 1}$ extreme when $x \in J$. The success of the argument when $p=m(m=2,3, \ldots)$ depends on Lemma 3.2 and the ability to 'capture' the term $(1-x)^{m}$ in the functions $H_{n}$ as shown in Theorem 3.7. When $p$ is not an integer, one probably needs a version of Lemma 3.2 involving partial sums of $(1-x)^{p}$.

Of course the ultimate goal is to precisely determine all extreme points for any $p$ and $q$. In conclusion, we make the following conjecture.

Conjecture 3.10. For fixed $p$ and $q$, there exists an angle $\widetilde{\theta}=\widetilde{\theta}(p, q)$ separating extreme points from nonextreme points as follows.

(a) If $0<p \leq 1$, then $\widetilde{\theta}=0, k_{x, 1}$ is extreme if $x \neq 1$ and $k_{1,1}$ is not extreme.

(b) If $p>1$, then $0<\widetilde{\theta}<\pi(p-1) /(p+1)$ and, for $x=\mathrm{e}^{i \theta}, k_{x, 1}$ is extreme if $\widetilde{\theta} \leq|\theta| \leq$ $\pi$ and $k_{x, 1}$ is not extreme if $0 \leq|\theta|<\widetilde{\theta}$.

In the special case $p=2, q=1$, further analysis suggests that the critical angle $\widetilde{\theta}$ should yield an extreme point. According to (ii) in Section 1 , for $0<p \leq 1$ the kernel function $k_{x, 1}$ is extreme if $x \neq 1$, independent of $q$. It is also well known that $k_{1,1}$ is not extreme for any $p$ and $q$, provided $q \geq p$. Thus part (a) of Conjecture 3.10 is correct if $0<p \leq 1$ and $q \geq p$. Recent work on identifying kernel functions that are not extreme shows that $k_{1,1}$ is not extreme if $q \geq 1$, independent of $p$. Moreover, if $q \geq 1$ and $p=m$ ( $m=2,3,4, \ldots)$, not only is $k_{1,1}$ not extreme but $k_{x, 1}$ is also not extreme for values of $x$ near 1. Paired with Corollary 3.9 we thus have extreme points corresponding to values of $x$ beyond the turning points and nonextreme points in intervals near the origin, providing strong evidence for the validity of part (b) of Conjecture 3.10, at least in the more tractable case of integer valued $p$. These nonextreme points results will be addressed in a planned companion paper.

\section{References}

[1] D. Aharonov and S. Friedland, 'On functions of bounded boundary rotation', Ann. Acad. Sci. Fenn. A I Math. 585 (1974), 3-18.

[2] E. Bishop, 'A minimal boundary for function algebras', Pacific J. Math. 9 (1959), 629-642.

[3] T. Gamelin, Uniform Algebras (Prentice Hall, Englewood Cliffs, NJ, 1969). 
[4] T. H. MacGregor, L. Brickman and D. R. Wilken, 'Convex hulls of some classical families of univalent functions', Trans. Amer. Math. Soc. 156 (1971), 91-107.

[5] T. H. MacGregor and D. R. Wilken, Handbook of Complex Analysis: Geometric Function Theory, Vol. 1 (North Holland/Elsevier, Amsterdam, 2002), 371-392.

[6] S. Perera, 'Support points and extreme points of some classes of analytic functions', PhD Thesis, State University of New York at Albany, 1983.

[7] J. Rice, 'Continuous linear functionals on compact and convex integral families of analytic functions', PhD Thesis, State University of New York at Albany, 1994.

KEIKO DOW, Canisius College, 2001 Main Street, Buffalo, NY 14208, USA

e-mail: dowk@canisius.edu

D. R. WILKEN, University At Albany, 1400 Washington Avenue, Albany, NY 12222, USA

e-mail: wilken@Math.Albany.edu 